\section{The transmission of AIDS}

Data that mothers can transmit HIV to infants through breast feeding add disturbing new insight into the mechanism of transmission.

IN Kigali, Rwanda, physicians have been following the development of infants born to 212 mothers who were seronegative for HIV (human immunodeficiency virus) at the time of delivery. "After a mean follow-up of 16.6 months, 16 of the 212 mothers became seropositive for HIV-1. Of their 16 infants, 9 became seropositive,' according to a paper in The New England Journal of Medicine (325, 593-598; 29 August 1991). Each of the infants was breast fed; mothers' milk was the route of HIV transmission.

These data add one more discouraging piece of evidence to the hypothesis that AIDS is more easily transmissible than people would like to believe. Although the idea that HIV might be transmitted through breast milk is not novel, the possibility of it ever happening has consistently been described as "rare" by AIDS researchers and epidemiologists. However, the seroconversion rate in Rwanda alone is in the range of 4.7 to 6.3 per cent a year, putting many children at risk. In the United States and Europe, where the virus is still not widespread among women, the data from Africa may not seem threatening. However, speculation that HIV may enter the heterosexual population (as it already has in Africa and Asia) needs to be taken seriously.

Thus it is crucial to know more about the mechanism of HIV transmission. At the seventh international conference on AIDS, held in Florence in June, new attention was paid to preliminary data showing that HIV may penetrate dendritic cells in genital (and possibly oral) mucosa. If this is true, then the belief that HIV transmission requires blood-to-blood contact falls on its face.

For the present, there is no suggestion that poor women in Rwanda or elsewhere should give up breast feeding, but that is mainly because they lack safe alternatives for feeding their infants. The dangers of non-sterile formula outweigh the statistical probability of contracting AIDS. But that is not the point.

The new data about mothers' milk merely adds to the urgency of learning more about mucosal transmission because the most likely explanation for HIV transmission through breast feeding is that the virus penetrates the mucosal lining of the gastrointestinal tract of infants. It may be necessary to abandon the comforting notion that HIV is extraordinarily difficult to transmit.

The unanswered questions about mucosal transmission are pertinent to a heated debate in the United States about the duties of HIV-infected medical professionals - particularly dentists and surgeons - who come into contact with patients' bodily fluids. The US government has asked professional groups to develop lists of procedures that HIV carriers or people with full-blown AIDS should avoid in medical or dental practice. It is a sensible, if conservative, approach to the unthinkable - contracting AIDS from one's doctor.

However, nearly 40 groups of health care professionals have declined to draw up guidelines on the grounds that the likelihood of doctor-patient transmission is so remote that guidelines are not necessary. It is true that the case of a Florida dentist who transmitted AIDS to a now-dying patient is the only known instance of such transmission. But it is also a matter of record that more than 6,000 health care workers are now known to be HIV-positive and the US Centers for Disease Control say that number probably is low.

The fact is that at present we do not know the full extent of the risk. But emerging data suggest that the recalcitrant groups of health professionals should think again.

\section{More genome ethics}

Now Baroness Warnock has joined the chorus saying that even knowledge can be ethically dangerous.

THE British Association for the Advancement of Science seems to have had a traditionally routine annual meeting at Plymouth last week. Industrialists complained that other industrialists were not spending enough on research and development, while environmentalists complained about all others but themselves. But something must be done about the way that meetings such as this are increasingly used to amplify the over-simple message that the human genome projects abound with ethical difficulties. At Plymouth, Baroness Warnock, the chairman of the committee whose largely sensible report became the basis for British legislation on human embryo research, was asking that there should now be legislation to restrict access to human genome data by insurance companies and employers.

What exactly are people afraid of? Insurance has been much discussed. Consider employment, and suppose that genome research some day describes an allelic version of a normal human gene that makes its carriers susceptible to the carcinogenic effect of a chemical used widely in some industrial process. What should happen then? Should plants using the process be shut down on the grounds that susceptible people might be employed in them? Or should employers be encouraged to discriminate among their potential employees, not hiring those discovered to be susceptible?

We do not have to wait for the outcome of the genome projects to know what will happen. There is an inherited susceptibility to vinyl chloride monomer, but the attempts by employers in the United States to avoid exposing susceptible people to danger have been met with a flurry of lawsuits. Does that imply that the right to work in the manufacture of PVC is one of the inalienable rights of man? Would it not be profitable to keep the plants going and to use part of the economic wealth created to compensate those with the bad luck to be susceptible? 